\title{
Microplastic analysis using chemical extraction followed by LC-UV analysis: a straightforward approach to determine PET content in environmental samples
}

\author{
Axel Müller ${ }^{1,2}$, Caroline Goedecke $^{1}$, Paul Eisentraut ${ }^{1}$, Christian Piechotta ${ }^{1}$ and Ulrike Braun ${ }^{1 *}$ (D
}

\begin{abstract}
Background: The ubiquitous occurrence of microplastic particles in marine and aquatic ecosystems was intensively investigated in the past decade. However, we know less about the presence, fate, and input paths of microplastic in terrestrial ecosystems. A possible entry path for microplastic into terrestrial ecosystems is the agricultural application of sewage sludge and solid bio-waste as fertilizers. Microplastic contained in sewage sludge also includes polyethylene terephthalate (PET), which could originate as fiber from textile products or as a fragment from packaging products (foils, bottles, etc.). Information about microplastic content in such environmental samples is limited yet, as most of the used analytical methods are very time-consuming, regarding sample preparation and detection, require sophisticated analytical tools and eventually need high user knowledge.
\end{abstract}

Results: Here, we present a simple, specific tool for the analysis of PET microplastic particles based on alkaline extraction of PET from the environmental matrix and subsequent determination of the monomers, terephthalic acid, using liquid chromatography with UV detection (LC-UV). The applicability of the method is shown for different types of PET in several soil-related, terrestrial environmental samples, e.g., soil, sediment, compost, fermentation residues, but also sewage sludge, suspended particles from urban water management systems, and indoor dust. Recoveries for model samples are between 94.5 and $107.1 \%$. Limit of determination and limit of quantification are absolute masses of 0.031 and $0.121 \mathrm{mg}$ PET, respectively. In order to verify the measured mass contents of the environmental samples, a method comparison with thermal extraction-desorption-gas chromatography-mass spectrometry (TED-GC/MS) was conducted. Both methods deliver similar results and corroborated each other. PET mass contents in environmental samples range from values below $L O Q$ in agriculture soil up to $57,000 \mathrm{mg} \mathrm{kg}^{-1}$ in dust samples.

Conclusions: We demonstrate the potential of an integral method based on chemical extraction for the determination of PET mass contents in solid environmental samples. The method was successfully applied to various matrices and may serve as an analytical tool for further investigations of PET-based microplastic in terrestrial ecosystems.

Keywords: Microplastic, Fibers, PET, Analysis, LC-UV, Soil, Sewage sludge, Depolymerization

*Correspondence: ulrike.braun@bam.de

1 Bundesanstalt für Materialforschung und -prüfung (BAM), Unter den Eichen 87, 12205 Berlin, Germany

Full list of author information is available at the end of the article

\section{Background}

Microplastic defined as plastic particles in the dimension of $1-1000 \mu \mathrm{m}[1]$ are considered a new emerging threat to ecosystems worldwide. Microplastic particles are observed in every environmental medium, however the effects on the ecosystem are still unclear. It is 
likely, however, that a further significant increase in the number of microplastic particles will have environmental impacts, whether it is a change in the accumulation behavior of natural particles [2] or the sorption and permeation of persistent pollutants of larger quantities [3, 4].

Marine ecosystems were intensively studied in the last decade, while the status of terrestrial ecosystems remains mainly unknown [5]. This knowledge gap is particularly alarming as plastic contamination in terrestrial ecosystems might be 4-23 times higher than in aquatic ecosystems [6].

Besides littering, a possible input path of plastic in soils is the application of sewage sludge as fertilizer [7]. Fibers and primary microplastic particles from laundry, cosmetic, and hygienic products are mostly retained in water treatment plants and end up in the sewage sludge [8]. Sewage sludge and municipal solid waste compost [9] are globally used as fertilizers in agriculture. A microplastic input of $63,000-430,000 \mathrm{t} \mathrm{a}^{-1}$ was estimated for European farmlands through the use of sludge as fertilizer [10]. Studies of agricultural soil detected concentrations between 78 particles $\mathrm{kg}^{-1}$ [11] and 18,760 particles $\mathrm{kg}^{-1}$ [12] and $0.88-0.95 \mathrm{mg} \mathrm{g}^{-1}$ [17]. Studies investigating the occurrence of microplastic in the soil after the applications of sewage sludge resulted in concentrations between 1100 and 3500 particles $\mathrm{kg}^{-1}$ [13]. Depending on the sewage sludge treatment process, sewage sludge shows concentration up to 34,000 particles $\mathrm{kg}^{-1}$ $[14,15]$, or mass content up to $12,000 \mathrm{mg} \mathrm{kg}^{-1}$ [16] or $3300 \mathrm{mg} \mathrm{kg}{ }^{-1}$ [17]. 92\% of the detected microplastic in soil aggregates from croplands in China consisted of synthetic fibers [12]. Synthetic fibers comprise $70 \%$ of polyethylene terephthalate (PET) regarding the production volume [18], and could remain up to 15 years in soils, after the application of sludge [19]. These fibers might negatively influence soil biota. A recent study indicate that the intake of PET fibers by snails leads to reduced food intake and induced damage in the villi of the gastrointestinal walls [20]. Furthermore, fibers might negatively affect the germination of ryegrass [21].

The few existing conducted studies about microplastic in soils use different analytical methods with non-comparable results. Therefore, relevant sources, occurrences, transport pathways, and the fate of microplastic are difficult to assess. Most studies use optical or microscopicspectroscopic methods as Fourier-transform infrared spectroscopy (FTIR) or Raman spectroscopy. These methods deliver information about particle numbers, size, and shape. Those spectroscopic methods are very time-consuming for environmental samples, especially because often, complex sample pretreatment is needed. It is mandatory to reduce the inorganic matrix and enrich the microplastic concentration before analysis [22, 23].
Usually, density separation is used for this purpose. To reduce the organic matrix before FTIR or microscopy analysis, an additional oxidation step with peroxide is needed [11, 12, 22, 24]. A faster, spectroscopic method is Fourier-transform near-infrared (FT-NIR) spectroscopy by the use of a fiber-optic reflection probe. It was successfully used to determine the presence of PET in soil samples without pretreatment, but requires minimum mass contents of $1 \%$, which is very high and practically not observed in reality [25].

An alternative is the use of integral methods, which are faster and deliver mass fractions, an important value for monitoring. Thermoanalytical methods determine polymer mass fractions by the detection of specific decomposition products formed under thermal treatment. Common are pyrolysis-gas chromatography-mass spectrometry (Py-GC/MS) [26, 27] or thermal extractiondesorption-gas chromatography-mass spectrometry (TED-GC/MS) [28, 29].

Furthermore, chemical methods are possible with two principal strategies. Either polymer chains can be extracted by solvents and detected afterward [17, 30, 31]. Regarding the poor solubility of polymers, including PET, these methods often require poisonous or expensive solvents. This is especially disadvantageous when large sample volumes must be analyzed, which is mandatory to account for the heterogeneity of soil samples [1]. The second principle is based on the decomposition of the polymeric chain and subsequent analysis of specific decomposition products. The latter is generally only possible when the polymer structure offers specific functionality for bond cut, which, for example, is present in polyesters or polyamides.

Regarding the analysis of suspected high contents of PET in soil from fertilization, such extraction techniques developed by Wang et al. [32] are of high interest for routine application. Wang et al. described a way to depolymerize PET in its monomers, among others, terephthalic acid, and to subsequently detect the terephthalic acid by LC-MS/MS (liquid chromatography coupled with tandem mass spectrometry). LC-based methods are widespread in analytical service laboratories, could be used in routine analysis, and have particularly low detection limits.

The objective of the present study is to adapt the sample extraction procedure described by Wang et al. to soil samples and to transfer the analysis to a LC-UV system (liquid chromatography with ultraviolet detection) [32]. LC-MS/MS is a complex method for the structure elucidation of trace substances in environmental samples, especially for chemical compounds of unknown composition (non-target analysis). The application of LC-UV, however, can only provide an assignable signal if the 
structure of the components is known. Since the terephthalic acid is already known as a target for the detection of PET, we have therefore adapted the LC-MS/ MS method by Wang et al. to LC-UV. LC-UV is a more robust and cost-effective method because the prerequisites for MS measurement (ultra-high vacuum) are not necessary, and the maintenance procedures for the MS, such as sticky char contaminations cannot occur. Here we present for the first time the use of LC-UV for the determination of PET mass content in soil. Such LC techniques require moderate conditions of sample preparation without critical chemicals in terms of costs and toxicity [17, 30, 31], and therefore allow investigations of high sample masses. This method is not limited to PET analysis in soil but suitable for the detection of PET particles in any solid environmental matrix, such as sewage sludge or bio-waste. It is also applicable for analysis of PET in dust or airborne particles and could even be used in the future for the monitoring analysis of PET fibers from washing machines or PET particles in bottled water.

\section{Materials and methods}

\section{Materials and environmental samples}

As a reference material bottle-grade PET pellets (PET Lighter C 93, provided by Equipolymers, Schkopau, Germany) were cryo-milled under constant cooling with liquid nitrogen in a Retsch CryoMill (Retsch GmbH, Haan, Germany). A pre-cooled 50-ml milling beaker equipped with a $25-\mathrm{mm}$ steel ball was used for milling. After milling at $5 \mathrm{~Hz}$ for $5 \mathrm{~min}$, the frequency was increased to $25 \mathrm{~Hz}$ for $5 \mathrm{~min}$ and subsequently reduced again to $5 \mathrm{~Hz}$ for $0.5 \mathrm{~min}$. Furthermore, additional real polymeric samples of PET were used. They came from everyday applications, such as fibers from textiles and fragments cut from a commercial single-use soda bottle.
For calibrations and recoveries experiments, a reference soil matrix, originating from a field test facility of the Bundesanstalt für Materialforschung und -pruefung was used. The used reference soil matrix came from a mostly plastic-free environment and was defined as sandy sand with $12 \%$ gravel, $70 \%$ Sand, $18 \%$ silt and clay, with a total organic carbon content of $0.7 \%$. As an alternative matrix, a mixture of $95 \%$ pure quartz sand and 5\% peat was used.

The environmental samples were chosen based on an expected PET mass content. All samples were collected in Germany and were air-dried. The selection of the samples did not aim to assess entry sources but to test the suitability of our method for real samples. An overview of the origin of the samples is given in Table 1. The table also includes the organic content of all samples, determined by the first heating of thermogravimetric analysis between 180 and $600{ }^{\circ} \mathrm{C}$ [33].

Beach sediments of $10 \mathrm{l}$ were sampled at the wash margin in Warnemuende, Germany. Here we expected possible microplastic particles from marine water. The agriculture soil sample was taken from a field in BadenWuerttemberg as a mixed sample of $2.5 \mathrm{l}$, consisting of 20 single samples collected with an Edelman-Driller. The agriculture soil sample originated from a field that was previously fertilized with sewage sludge.

We suspected that bio-waste may have a high percentage of littering content from plastic packaging. Compost samples were provided by a compost plant in Lower Saxony. The samples A, B, and C are taken at different days, respectively. All compost samples were cryo-milled (Retsch GmbH, Haan, Germany). Furthermore, fermentation residue samples were taken from a biogas plant in Berlin. Sample A was from the aerobization container, sample B was a liquid fermentation residue, and sample $C$ a solid fermentation residue. Fermentation residues were

Table 1 Overview of the environmental samples

\begin{tabular}{|c|c|c|c|}
\hline Environmental sample & Origin & Presumed source of PET entry & $\begin{array}{l}\text { Pyrolisable organic } \\
\text { content }(180- \\
\left.600^{\circ} \mathrm{C}\right)\end{array}$ \\
\hline Beach sediment & $\begin{array}{l}\text { Warnemuende } \\
54.194910 \text { N } 12.140777 \mathrm{E}\end{array}$ & Marine littering & $<1 \%$ \\
\hline Agriculture soil & $\begin{array}{l}\text { Baden-Wuerttemberg } \\
48.787672 \text { N 8.179543 E }\end{array}$ & Performed sewage sludge fertilization & $3.6 \%$ \\
\hline Compost & Lower Saxony & Littering from urban waste & $18-24 \%$ \\
\hline Fermentation residue & Berlin & Littering from urban waste & $34-39 \%$ \\
\hline Suspended particles of WWTP effluent & Kaiserslautern & No content suspected due to WWTP process & $59 \%$ \\
\hline Filter residue of greywater & Rhineland-Palatinate & Fibers of textile washing & $62 \%$ \\
\hline Sewage sludge & Berlin & Separation and enrichment due to WWTP process & $47 \%$ \\
\hline Dust samples & Berlin & Textile fibers in indoor air & $72-78 \%$ \\
\hline
\end{tabular}


homogenized by milling in a liquid nitrogen-cooled centrifugal mill (ZM 200, Retsch GmbH, Haan, Germany) with 1-mm mesh size.

Although the paper focuses on soils, we investigated also samples from urban water management. Here we expected PET, especially from fragmented littering or from textile products (fibers). Suspended particles were collected using a sedimentation trap in the effluent of a wastewater treatment plant (WWTP) in Kaiserslautern. Here we did not expect a PET signal from the purification line of the plant. Particles from greywater were collected by filtration $(>100 \mu \mathrm{m})$, from a farmstead in Rhineland-Palatinate. Sewage sludge samples were taken from a WWTP in Berlin.

Finally, we chose two indoor dust samples with an expected high loading of textile fibers. Dust samples were collected in two households in Berlin.

For sample preparation butanol ( $>99.5 \%)$, methanol (>99.9\%), hydrochloric acid (analytical grade), and potassium hydroxide (analytical grade) were obtained from Merck-Schuchardt (Hohenbrunn, Germany). SPE cartridges, type OASIS VAC HLB 6 cc with $200 \mathrm{mg}$ solid phase and $30 \mu \mathrm{m}$ particle size, were from Waters (Eschborn, Germany). Glass fiber filters, type 9 , with $90 \mathrm{~mm}$ diameter and syringe filters based on cellulose acetate with a pore size of $0.45 \mu \mathrm{m}$ were both purchased from TH Geyer (Berlin, Germany).

\section{LC-UV}

LC-UV analysis was carried out on an HP-1100 system (Agilent Technologies, Santa Clara, California, USA), equipped with a binary pump (G1312A), autosampler (G1313A), thermostatted column compartment and diode array detector (1315A). The system was equipped with an accucore reversed phase column $(100 \mathrm{~mm} \times 2.1 \mathrm{~mm}$; particle size $2.6 \mu \mathrm{m}$; Thermo Fisher Scientific, Waltham, Massachusetts, USA). The column oven was set to $35{ }^{\circ} \mathrm{C}$. The injection volume was $50 \mu \mathrm{l}$. The eluent flow was set to $200 \mu \mathrm{L} \mathrm{min}{ }^{-1}$. Mobile phases were ultrapure water with $1 \%$ acetic acid (Solvent A) and $1 / 1 \mathrm{v} / \mathrm{v}$ methanol/acetonitrile (Solvent B). The starting gradient was $95 \% \mathrm{~A}$ at $0 \mathrm{~min}, 80 \% \mathrm{~A}$ at $7 \mathrm{~min}, 80 \%$ $\mathrm{A}$ at $15 \mathrm{~min}, 95 \% \mathrm{~A}$ at $20 \mathrm{~min}$ and $95 \% \mathrm{~A}$ at $30 \mathrm{~min}$. The LC system was operated with a UV-detector at a wavelength of $240 \mathrm{~nm}$. At this wavelength, the UV/Vis spectra of terephthalic acid own a broad absorption peak [34]. Chemstation (A.09.03 version, Agilent Technologies, Santa Clara, California, USA) was used for data acquisition, processing, and peak integration.

\section{TED-GC/MS}

To check whether the method provides reliable and comparable results, environmental samples were additionally measured with a thermal analytical method. Thermal extraction-desorption-gas chromatography-mass spectrometry (TED-GC/MS) was used as a reference method. Measurements were conducted as described by Eisentraut et al. [28, 29]. In routine analysis, sample masses of $20 \mathrm{mg}$ (composts, fermentation residue) were analyzed and variable masses between 6 and $15 \mathrm{mg}$ were analyzed for samples from water and air (samples from WWTP, dust). In TED-GC/MS, ethyl benzoate was used as a specific marker for PET ( $\mathrm{m} / \mathrm{z}$ value of 105$)$.

\section{Results and discussion \\ Optimization of the extraction process for terephthalic acid}

The sample extraction was performed in principle on the procedure described by Wang et al. [32]. However, according to the different detection systems and different samples, several steps were simplified and adapted to higher sample masses. In general, $20 \mathrm{~g}$ of sample was used, but sample mass varied for other matrices depending on expected PET content and available sample mass. $20 \mathrm{ml}$ of 1-butanol was added to the solid sample in a $100 \mathrm{ml}$ round flask. $1 \mathrm{~g}$ of potassium hydroxide pellets were added. The mixture was heated to $115{ }^{\circ} \mathrm{C}$ in an oil bath under constant stirring for $1 \mathrm{~h}$. A tubular cooler was used for reflux. $50 \mathrm{ml}$ of ultra-pure water was added, and the 2-phase system was mixed for $1 \mathrm{~h}$ on a stirring plate at $300 \mathrm{rpm}$. The extract was then vacuum filtrated with a glass fiber filter to remove the sediment/solids and collected. The buoyant organic phase was removed with a pipet. $10 \mathrm{ml}$ of the aqueous phase was transferred to a $100 \mathrm{ml}$ flask using a graduated pipette. The solution was diluted 1:10 with ultra-pure water, and the $\mathrm{pH}$ was adjusted to 2.5 by adding hydrochloric acid (conc. $=10 \%$ ).

Before the LC-UV measurements, the aqueous extracts were cleaned up with a solid-phase extraction to avoid interferences of the organic matrix during analysis. A constant vacuum of 0.6 bar was applied using a vacuum chamber connected to a membrane pump. Cartridges were subsequently equilibrated and conditioned with $10 \mathrm{ml}$ methanol and $10 \mathrm{ml}$ ultra-pure water, respectively. Afterward, $10 \mathrm{ml}$ of the aqueous extracts were passed over the cartridge. $5 \mathrm{ml}$ of a 90:10 ultra-pure water/ methanol mixture was used for washing the cartridges, before elution of the analyte with $10 \mathrm{ml}$ methanol. The eluate was collected in $20 \mathrm{ml}$ screw cap vials, and the solvent got evaporated. Precipitated terephthalic acid was subsequently reconstituted in $10 \mathrm{ml}$ ultra-pure water, of which $1 \mathrm{ml}$ was filtrated with a $0.45 \mu \mathrm{m}$ syringe filter and transferred to LC-vials with PTFE-lined screw caps. The sample was acidified to a $\mathrm{pH}$ of $1-1.5$ by adding $25 \mu \mathrm{HCl}$ (conc. $=1 \%$ ). Acidification ensures complete protonation of the terephthalic acid. Pre-experiments showed a 
sharpened peak form in the chromatogram and increased analytical sensitivity after acidification.

\section{Determination of the quality parameters}

A 6-level calibration with concentrations from 0 to $269 \mathrm{mg}$ PET kg${ }^{-1}$ in the reference soil was prepared, following the described sample preparation. Therefore, reference soil samples of $20 \mathrm{~g}$ were spiked with PET particles, which originated from cryo-milled PET pellets. All concentration levels were prepared in duplicates. All values were based on triplicate measurements. By using reference measurements, the peak in the chromatogram with a retention time of $7.7 \mathrm{~min}$ at a wavelength of $240 \mathrm{~nm}$ was identified as terephthalic acid (Fig. 1).

Figure 2 shows the linear relationship between the initially weighed PET and the signal for terephthalic acid.

The coefficient of determination was found to be $R^{2}>0.998$. Linearity was given up to $300 \mathrm{mg}$ PET kg-1 reference soil and defined the working range. This maximum mass content of $300 \mathrm{mg}$ PET kg-1 corresponded to a concentration of $12 \mathrm{mg} \mathrm{l}^{-1}$ terephthalic acid in the analyzed aqueous phase. The maximum solubility of terephthalic acid in water was $15 \mathrm{mg} \mathrm{l}^{-1}$ at $\mathrm{pH} 7$ and $25^{\circ} \mathrm{C}$ [35]. The measurement uncertainty based on a triplicate measurement was $<0.5 \%$. According to DIN 32645, with a significance level of 0.9 , the calculated limit of determination (LOD) and limit of quantification (LOQ) were $0.031 \mathrm{mg}$ and $0.121 \mathrm{mg}$ absolute PET, respectively. Based on the conducted calibration with $20 \mathrm{~g}$ reference soil matrix, this corresponds to a LOD of $1.55 \mathrm{mg} \mathrm{kg}^{-1}$ and LOQ of $6.05 \mathrm{mg} \mathrm{kg}^{-1}$. The determined LOQ and LOD

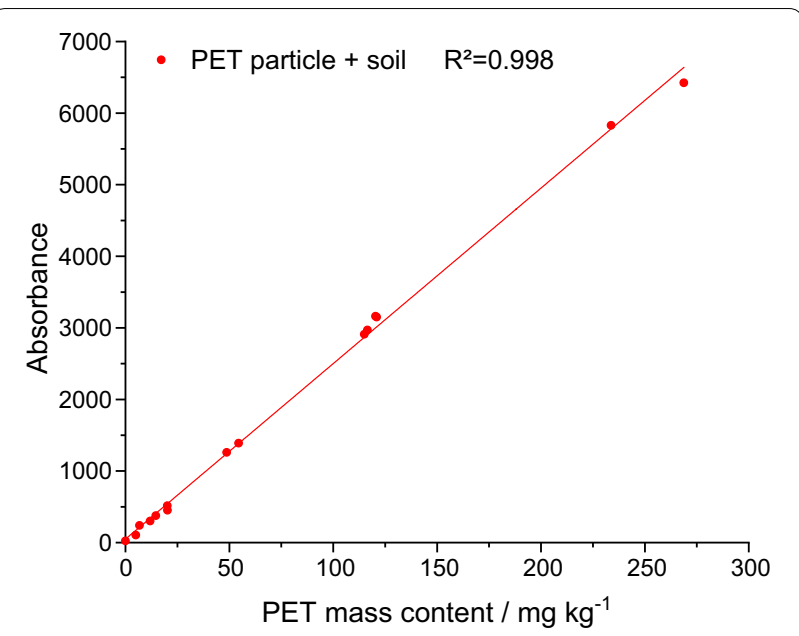

Fig. 2 Calibration slope for PET-spiked reference soil samples measured by LC-UV. PET mass refers to the initial spiked PET mass. All measurements were performed as triplicates

were specific for the used reference soil matrix and can be varying for other matrices. The calculated LOQ of $6.05 \mathrm{mg} \mathrm{kg}^{-1}$ PET of our developed method was significantly higher, compared to Wang et al., they calculated a LOQ of $53.0 \mu \mathrm{g} \mathrm{kg}^{-1}$ PET. This LOQ was two magnitudes lower than our LOQ, mainly due to the use of different detectors. However, it is noteworthy that the calculated LOQ in our study was not based on a calibration using water and purified terephthalic acid, but on a calibration based on the initially spiked PET in a reference soil matrix.
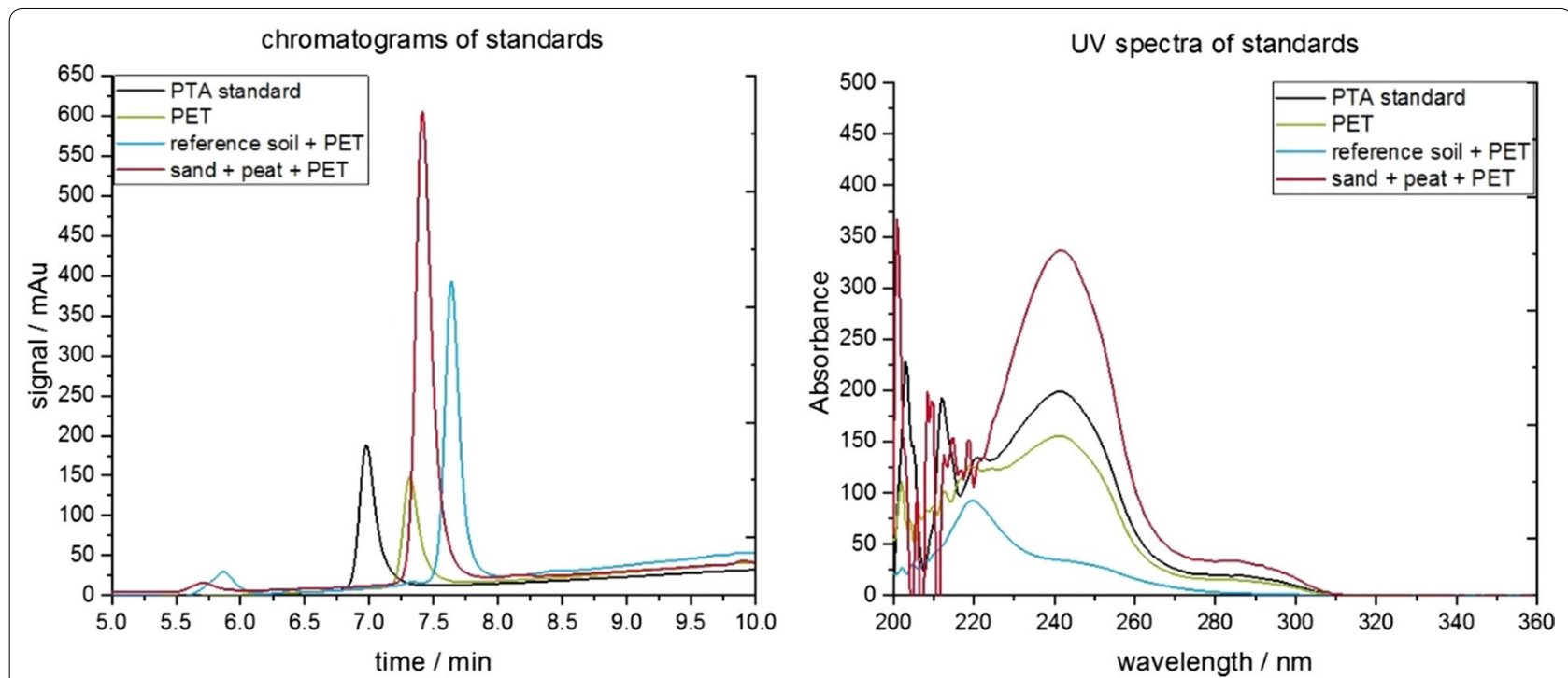

Fig. 1 Exemplary chosen chromatograms (left) and UV-spectra (right) of terephthalic acid (PTA) standard solution and extracts of PET, PET-spiked reference soil, and PET-spiked sand with peat 
To achieve a high level of quality control, control experiments were conducted before each series of measurements to test the measurement quality of the LC-UV system. These were measurements of a terephthalic acid solution as well as repeated measurements of calibration standards of reference soil with different PET contents. Additionally, blanks of purified water were measured after environmental samples and showed no carry-overs of terephthalic acid or organic matrix compounds.

Table 2 summarizes the results of different model matrices containing different spiked PET types. We selected two different mass contents for all sample sorts, which covered the linear calibration area of the method as well as a compromise of microplastic contents in existing works [12, 16, 17]. Recoveries were based on

Table 2 Recovery rates of different PET types in model samples, with two different spiking levels

\begin{tabular}{llc}
\hline Model sample & $\begin{array}{l}\text { Spiked mass } \\
\text { contents } / \mathbf{m g ~ k g}^{-\mathbf{1}}\end{array}$ & $\begin{array}{l}\text { Recovery } \\
\text { rate*/\% }\end{array}$ \\
\hline Reference soil + bottle fragments & 213 & 97.3 \\
& 431 & 94.8 \\
Reference soil + fabric fibers & 139 & 101.9 \\
& 191 & 98.5 \\
Sand + 5\% peat + PET particles & 210 & 107.1 \\
& 475 & 94.5 \\
\hline
\end{tabular}

Measurements were performed as duplicate reference soil calibration, as shown in Fig. 2. Sample masses of $20 \mathrm{~g}$ were analyzed in all cases.

Calculated recoveries for the sand and peat matrix were 94.5 and $107.1 \%$ and proofed the robustness of the method and the applicability for different environmental matrices. Calculated recoveries for different PET types, were 97.3 and $94.8 \%$ for bottle fragments, and 101.9 and $98.5 \%$ for fabric fibers. Generally, lower recoveries for the samples spiked with higher PET masses was explained by the limited solubility of terephthalic acid in water. Interference of the solid organic matter of the matrix on the detection of the terephthalic acid was not evident, as recoveries in the sand-peat matrix were not affected by the higher solid organic matter content compared to the reference soil. Hence, the method applied to different PET types and different soil matrices.

\section{Analysis of environmental samples}

In the next step, real environmental samples were analyzed. The values of PET mass contents were based on extraction as described above and subsequent duplicate or triplicate measurement. Table 3 shows the detected mass contents and the extracted sample mass, which differed from 150 to $300 \mathrm{mg}$ for dust samples up to 10,000-50,000 mg for sewage sludge, agriculture soil, and sediment samples. Figure 3 presents exemplary chromatograms and UV-spectra of selected samples.

PET was found in all investigated environmental samples, except for the samples of beach sediment.

Table 3 Detected PET mass contents in environmental samples using PET extraction and TED-GC/MS

\begin{tabular}{|c|c|c|c|c|}
\hline \multirow[t]{2}{*}{ Environmental sample } & \multicolumn{2}{|l|}{ PET extraction } & \multicolumn{2}{|l|}{ TED-GC/MS } \\
\hline & Sample weight/mg & Mass content/mg kg ${ }^{-1}$ & Sample weight/mg & $\begin{array}{l}\text { Mass } \\
\text { content/ } \\
\mathrm{mg} \mathrm{kg}^{-1}\end{array}$ \\
\hline Beach sediment, 1. extraction & 50,000 & Below LOD & - & - \\
\hline Beach sediment, 2. extraction & 50,000 & Below LOD & - & - \\
\hline Agriculture soil, 1. extraction & 20,000 & Below LOQ & - & - \\
\hline Agriculture soil, 2. extraction & 20,000 & Below LOQ & - & - \\
\hline Compost A & 5000 & 81.5 & 21.3 & Below LOD \\
\hline Compost B & 5000 & Below LOQ & 23.9 & Below LOD \\
\hline Compost C & 5000 & 102 & 22.1 & Below LOD \\
\hline Fermentation residue A & 3000 & 777 & 20.4 & Below LOD \\
\hline Fermentation residue B & 3000 & 210 & 20.7 & Below LOD \\
\hline Fermentation residue $\mathrm{C}$ & 3000 & 475 & 20.5 & Below LOD \\
\hline Particles of WWTP effluent & 5000 & 38.6 & 14.9 & Below LOD \\
\hline Filter residue of greywater & 500 & 1430 & 9.87 & Below LOQ \\
\hline Sewage sludge, 1. extraction & 10,000 & 98.7 & - & - \\
\hline Sewage sludge, 2. extraction & 10,000 & 142 & - & - \\
\hline Dust sample A & 276 & 12,500 & 6.11 & 7100 \\
\hline Dust sample B & 149 & 43,600 & 8.23 & 57,000 \\
\hline
\end{tabular}



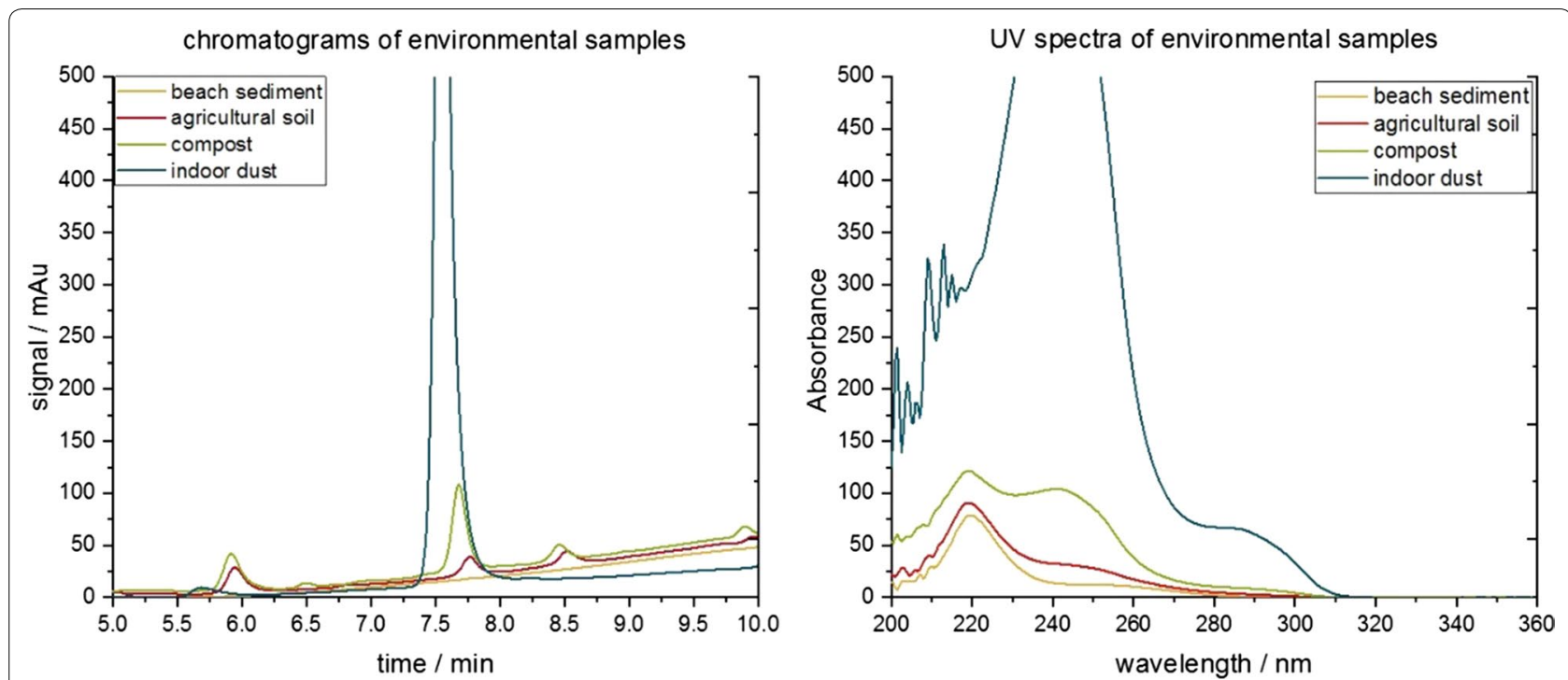

Fig. 3 Exemplary chosen chromatograms (left) and UV-spectra (right) of selected environmental samples with varying organic content and PET content

Lowest PET mass contents of 3.85 and $3.99 \mathrm{mg} \mathrm{kg}^{-1}$ were detected in the agriculture soil samples, however, this is below LOQ.

PET mass contents of compost samples were between 10.2 (below LOQ) and $102 \mathrm{mg} \mathrm{kg}^{-1}$. Compared to the compost, fermentation residues showed all over higher mass contents with values up to $776 \mathrm{mg} \mathrm{kg}^{-1}$ in the sample from the aerobization container. Mass contents in the liquid and solid fermentation residues were 209 and $475 \mathrm{mg} \mathrm{kg}^{-1}$, respectively. A correlation between the organic content and the observed PET findings could not be found for individual samples.

In the urban water management samples, an even higher PET content of $1430 \mathrm{mg} \mathrm{kg}^{-1}$ was found in the filter residue of greywater. The investigated sewage sludge showed mass contents of 98.7 and $141 \mathrm{mg} \mathrm{kg}^{-1}$, while only $38.6 \mathrm{mg} \mathrm{kg}^{-1}$ PET was found in the suspended particles of the WWTP effluent.

The highest PET mass contents of 12,500 and $57,000 \mathrm{mg} \mathrm{kg}^{-1}$ were found in the indoor dust samples. The determined polymer type was congruent with the findings of Lui [36] and Vianello [37] and suggests a future application in the field of dust analysis.

Some of the most promising samples were analyzed in parallel using TED-GC/MS. It should be noticed, that all experiments run with lower samples masses. The results were also summarized in Table 3. The findings of biowaste samples were all below LOD. Additional investigation using TED-GC/MS of samples with high PET findings from urban waste management did also not result is significant signals. However, PET was clearly found in the dust samples with amounts of $7100 \mathrm{mg} \mathrm{kg}-1$ and $57,000 \mathrm{mg} \mathrm{kg}^{-1}$. The results of LC-UV and TED-GC/ MS corroborated each other.

Using TED-GC/MS the LOD for PET was $0.88 \mu \mathrm{g}$ absolute (fivefold signal-to-noise ratio). In environmental samples the variability of this value is influenced by inherent matrix components and LOD values of up to $1.37 \mu \mathrm{g}$ were detected (Kittner et al. 2020, unpublished data). The determination of LOQ for TED-GC/MS according to DIN 32645 was not possible, because the standard deviation was found to change with relative PET content in the environmental matrix.

If the values of the PET extraction were extrapolated to the masses used for the TED-GC/MS measurement (clearly less, namely $20.4 \mathrm{mg}$ and $9.87 \mathrm{mg}$ ) a signal can only be expected for the fermentation residues and the greywater sample. Whereas in TED-GC/MS measurement the result of greywater indicates a minor content of PET, no evidence of any PET presence was found in the fermentation residue samples.

Different reasons might be responsible for missing PET signal in the TED-GC/MS measurements of the fermentation residue samples. First, the organic environmental matrix influenced the formation of PET marker for LC-UV measurement stronger than expected from previous measurements. However, such a correlation to the background value related to the organic content in those samples (see Table 1) could not be determined. Second, the PET extraction procedure did not discriminate different kinds of polyesters, such as polyethylene terephthalate, polybutylene terephthalate or polybutylene adipate 
terephthalate. The latter is a biodegradable polymer that might be present in larger quantities in fertilizer samples from urban waste and could, therefore, be responsible for the high terephthalic acid findings in LC-UV. In contrast to that, TED-GC/MS detected ethyl benzoate as a specific marker, which could only be formed by the decomposition of PET. However, such a reason was not plausible for the greywater sample. In fact, there could also be a third, and this could be the main reason for explaining these differences. Terrestrial samples own a high heterogeneity, and $20 \mathrm{mg}$ of sample in TED-GC/ MS measurement might be too low for a representative measurement. For the chemical extraction method, the used extracted samples masses were always higher and will, therefore, catch a higher number of representative particles.

Even if there were still uncertainties about background signals, contributions of possible false-positive signals from other polymers, or the representatives of the analyzed samples, it became clear that a relative classification of the signal intensities of both methods was possible.

\section{Conclusions}

The goal of this study was to adapt and further simplify the chemical extraction of PET, proposed by Wang et al. [32]. It was optimized for higher soil sample masses and transferred to an LC-UV system. LC-UV is commonly available in routine labs, no poisonous chemicals were needed when larger sample volumes must be analyzed (regarding the representativeness of the sample), it was easy to automatize, a measurement took only $30 \mathrm{~min}$ and evaluation of the data did not need any high user knowledge.

Within this study, we showed the application of this new LC-UV analysis, including optimized sample preparation for the determination of PET in environmental samples. The recovery of spiked samples was between 95 and 107\%, using different PET sorts and soil types. The sample size was up to $50 \mathrm{~g}$ and can be adjusted easily to even higher masses. This is of high interest for routine analysis of soils in future, because this would avoid additional sample preparation steps for analysis (e.g., density separation). A LOD and LOQ was determined of $0.031 \mathrm{mg}$ and $0.121 \mathrm{mg}$ absolute PET, respectively.

Using LC-UV, PET signals was found in all environmental samples: terrestrial samples, bio-waste residues, urban wastewater, and indoor dust. Mass contents varied strongly dependent on the sample between values below LOQ in agricultural soil and $57,000 \mathrm{mg} \mathrm{kg}^{-1}$ in indoor dust. Values for sewage sludge, compost, and fermentation residue were up to three magnitudes higher than in agricultural soil. Therefore, fertilization with the latter could be a possible source for microplastic in agroecosystems.

The proposed extraction can be used to quantify the mass input of PET into agroecosystems, thus providing a better understanding of the microplastic input by fertilization with sewage sludge and bio-waste. Advantageous of the method is the applicability for complex matrices. The method is a cost-effective and simple integral method and applicable for diverse environmental matrices.

The present method is a useful addition in the field of microplastic analysis and could be used for the analysis of PET in samples, but also where high findings expected. This could be PET particles in bottled water or samples with a high load of especially PET fibers, e.g., indoor dust samples or washing machine effluent. However, it should be noticed, that for environmental samples in general (e.g., like surface water, air samples) with more complex mixtures of different MP particles other method are more suitable.

Finally, this work demonstrates that also simple methods can be applied for specific questions of microplastic analytics. The use of such alternatives depends on the scientific issue of the analysis, i.e., the aim of the investigation. The present method, but also TED-GC/MS determine mass contents and are not comparable with methods for determining particle numbers, which can provide an even more in-depth description of microplastic (including particle shape and size). However, the described methods aim to be faster applicable (including sample preparation and detection) and less complicated in use. This is the only way to perform comprehensive measurements, e.g., for the screening of hot-spot entries.

However, in the end it will always be the individual goal of an investigation campaign, which investigation result should be achieved with which time horizon and analytical depth.

\section{Abbreviations}

FTIR: Fourier-transform Infrared spectroscopy; FT-NIR: Fourier-transform nearinfrared spectroscopy; LC-MS/MS: Liquid chromatography-tandem mass spectrometry; LC-UV: Liquid chromatography with UV-detection; LOD: Limit of detection; LOQ: Limit of quantification; PET: Polyethylene terephthalate; PTFE: Polytetrafluoroethylene; Py-GC-MS: Pyrolysis-gas chromatography-mass spectrometry; rpm: Revolutions per minute; TED-GC/MS: Thermal extractiondesorption-gas chromatography-mass spectrometry.

\section{Acknowledgements}

We are grateful to our colleagues Dominique Lörchner and Robert Köppen for their support with liquid chromatography measurements as well as FranzGeorg Simon for providing the sewage sludge samples. We kindly thank the group of Heidrun Steinmetz from the Technical University of Kaiserslautern, making samples of WWTP effluent and greywater available. Furthermore, we thank Claus Gerhard Bannick for the compost and fermentation residues samples, Katrin Scholz and David Kaiser (all from German Environment Agency, Germany) as well as Henner Hollert (Goethe University Frankfurt, Germany) for 
meaningful discussions. A very special thanks goes finally to Maria Kittner and Yosri Wiesner.

\section{Authors' contributions}

$A M, C G$, and UB designed the experimental set-up. Sample extractions were carried out by AM. AM, CG, and CP were responsible for development of the LC method. AM performed LC measurements and data acquisition and processing. PE conducted TED-GC/MS measurements. AM and UB drafted the manuscript. All authors edited and reviewed the manuscript. All authors read and approved the final manuscript.

\section{Funding}

This study was part of the Projekt "Plastik in Böden", FKZ 371772 2320, funded by the German Enviroment Agency, Germany.

\section{Availability of data and materials}

Material and data are described in the main manuscript. The datasets used and/or analyzed during the current study are available from the corresponding author on reasonable request.

\section{Ethics approval and consent to participate}

Not applicable.

\section{Consent for publication}

Not applicable.

\section{Competing interests}

The authors declare that they have no competing interests.

\section{Author details}

'Bundesanstalt für Materialforschung und -prüfung (BAM), Unter den Eichen 87, 12205 Berlin, Germany. ${ }^{2}$ RWTH Aachen University, Worringer Weg 1, 52062 Aachen, Germany.

Received: 26 February 2020 Accepted: 20 May 2020

\section{Published online: 02 June 2020}

\section{References}

1. Technical Report ISO/PRF TR 21960. (2019-in progress) Plastics-Environmental aspects-State of knowledge and methodologies, ISO-International Organization of Standardization

2. Porter A, Lyons BP, Galloway TS, Lewis C (2018) Role of marine snows in microplastic fate and bioavailability. Environ Sci Technol 52(12):7111-7119

3. Wang F, Wong CS, Chen D, Lu X, Wang F, Zeng EY (2018) Interaction of toxic chemicals with microplastics: a critical review. Water Res 139:208-219

4. Müller A, Becker R, Dorgerloh U, Simon F-G, Braun U (2018) The effect of polymer aging on the uptake of fuel aromatics and ethers by microplastics. Environ Pollut 240:639-646

5. He DF, Luo YM, Lu SB, Liu MT, Song Y, Lei LL (2018) Microplastics in soils: analytical methods, pollution characteristics and ecological risks. TracTrend Anal Chem 109:163-172

6. Horton AA, Walton A, Spurgeon DJ, Lahive E, Svendsen C (2017) Microplastics in freshwater and terrestrial environments: evaluating the current understanding to identify the knowledge gaps and future research priorities. Sci Total Environ 586:127-141

7. Weithmann N, Möller JN, Löder MGJ, Piehl S, Laforsch C, Freitag R (2018) Organic fertilizer as a vehicle for the entry of microplastic into the environment. Sci Adv 4(4):8060

8. Carr SA, Liu J, Tesoro AG (2016) Transport and fate of microplastic particles in wastewater treatment plants. Water Res 91:174-182

9. Watteau F, Dignac M-F, Bouchard A, Revallier A, Houot S (2018) Microplastic detection in soil amended with municipal solid waste composts as revealed by transmission electronic microscopy and pyrolysis/GC/MS Front Sustain Food Syst 2:81

10. Nizzetto L, Futter M, Langaas S (2016) Are agricultural soils dumps for microplastics of urban origin? Environ Sci Technol 50(20):10777-10779
11. Liu M, Lu S, Song Y, Lei L, Hu J, Lv W, Zhou W, Cao C, Shi H, Yang X (2018) Microplastic and mesoplastic pollution in farmland soils in suburbs of Shanghai, China. Environ Pollut 242:855-862

12. Zhang G, Liu Y (2018) The distribution of microplastics in soil aggregate fractions in southwestern China. Sci Total Environ 642:12-20

13. Corradini F, Meza P, Eguiluz R, Casado F, Huerta-Lwanga E, Geissen V (2019) Evidence of microplastic accumulation in agricultural soils from sewage sludge disposal. Sci Total Environ 671:411-420

14. Mahon AM, O'Connell B, Healy MG, O'Connor I, Officer R, Nash R, Morrison $L$ (2017) Microplastics in sewage sludge: effects of treatment. Environ Sci Technol 51(2):810-818

15. Corradini F, Bartholomeus H, Huerta Lwanga E, Gertsen H, Geissen V (2019) Predicting soil microplastic concentration using vis-NIR spectroscopy. Sci Total Environ 650:922-932

16. Zhang J, Wang L, Halden RU, Kannan K (2019) Polyethylene terephthalate and polycarbonate microplastics in sewage sludge collected from the United States. Environ Sci Technol Lett 6(11):650-655

17. Dierkes G, Lauschke T, Becher S, Schumacher H, Földi C, Ternes T (2019) Quantification of microplastics in environmental samples via pressurized liquid extraction and pyrolysis-gas chromatography. Anal Bioanal Chem 411(26):6959-6968

18. Geyer R, Jambeck JR, Law KL (2017) Production, use, and fate of all plastics ever made. Sci Adv 3(7):e1700782

19. Zubris KAV, Richards BK (2005) Synthetic fibers as an indicator of land application of sludge. Environ Pollut 138(2):201-211

20. Song Y, Cao C, Qiu R, Hu J, Liu M, Lu S, Shi H, Raley-Susman KM, He D (2019) Uptake and adverse effects of polyethylene terephthalate microplastics fibers on terrestrial snails (Achatina fulica) after soil exposure. Environ Pollut 250:447-455

21. Boots B, Russell CW, Green DS (2019) Effects of microplastics in soil ecosystems: above and below ground. Environ Sci Technol 53(19):11496-11506

22. Scheurer M, Bigalke M (2018) Microplastics in swiss floodplain soils Environ Sci Technol 52(6):3591-3598

23. Hurley R, Woodward J, Rothwell JJ (2018) Microplastic contamination of river beds significantly reduced by catchment-wide flooding. Nat Geosci 11(4):251-257

24. Piehl S, Leibner A, Loder MGJ, Dris R, Bogner C, Laforsch C (2018) Identification and quantification of macro- and microplastics on an agricultural farmland. Sci Rep 8(1):17950

25. Paul A, Wander L, Becker R, Goedecke C, Braun U (2019) High-throughput NIR spectroscopic (NIRS) detection of microplastics in soil. Environ Sci Pollut Res Int 26(8):7364-7374

26. Fischer M, Scholz-Böttcher BM (2017) Simultaneous trace identification and quantification of common types of microplastics in environmental samples by pyrolysis-gas chromatography-mass spectrometry. Environ Sci Technol 51(9):5052-5060

27. Fries E, Dekiff JH, Willmeyer J, Nuelle MT, Ebert M, Remy D (2013) Identification of polymer types and additives in marine microplastic particles using pyrolysis-GC/MS and scanning electron microscopy. Environ Sci Process Impacts 15(10):1949-1956

28. Dümichen E, Eisentraut P, Bannick CG, Barthel AK, Senz R, Braun U (2017) Fast identification of microplastics in complex environmental samples by a thermal degradation method. Chemosphere 174:572-584

29. Eisentraut $P$, Dümichen $E$, Ruhl AS, Jekel M, Albrecht M, Gehde M, Braun U (2018) Two birds with one stone - fast and simultaneous analysis of microplastics: microparticles derived from thermoplastics and tire wear. Environ Sci Technol Lett 5(10):608-613

30. Fuller S, Gautam A (2016) A procedure for measuring microplastics using pressurized fluid extraction. Environ Sci Technol 50(11):5774-5780

31. Elert AM, Becker R, Duemichen E, Eisentraut P, Falkenhagen J, Sturm $\mathrm{H}$, Braun U (2017) Comparison of different methods for MP detection: what can we learn from them, and why asking the right question before measurements matters? Environ Pollut 231(Pt 2):1256-1264

32. Wang L, Zhang JJ, Hou SG, Sun HW (2017) A simple method for quantifying polycarbonate and polyethylene terephthalate microplastics in environmental samples by liquid chromatography-tandem mass spectrometry. Environ Sci Technol Lett 4(12):530-534

33. Dittmann D, Braun U, Jekel M, Ruhl AS (2018) Quantification and characterisation of activated carbon in activated sludge by thermogravimetric and evolved gas analyses. J Environ Chem Eng 6(2):2222-2231 
34. NIST Chemistry WebBook. https://webbook.nist.gov/chemistry. Accessed 20 Apr 2020

35. Merck (2011), Terephthalsäure zur Synthese. Sicherheitsdatenblatt (Version 2.3)

36. Liu C, Li J, Zhang Y, Wang L, Deng J, Gao Y, Yu L, Zhang J, Sun H (2019) Widespread distribution of PET and PC microplastics in dust in urban China and their estimated human exposure. Environ Int 128:116-124

37. Vianello A, Jensen RL, Liu L, Vollertsen J (2019) Simulating human exposure to indoor airborne microplastics using a Breathing Thermal Manikin. Sci Rep 9(1):8670

\section{Publisher's Note}

Springer Nature remains neutral with regard to jurisdictional claims in published maps and institutional affiliations.
Submit your manuscript to a SpringerOpen ${ }^{0}$ journal and benefit from:

- Convenient online submission

- Rigorous peer review

- Open access: articles freely available online

- High visibility within the field

- Retaining the copyright to your article

Submit your next manuscript at $\boldsymbol{\nabla}$ springeropen.com 